\title{
Study protocol for a randomised controlled trial of CBT vs antipsychotics vs both in 14- 18-year-olds: Managing Adolescent first episode Psychosis: a feasibility study (MAPS)
}

Melissa Pyle ${ }^{1,2}$, Matthew R. Broome ${ }^{3,4,5,6^{*}}$, Emmeline Joyce ${ }^{1}$, Graeme MacLennan ${ }^{7}$, John Norrie ${ }^{8}$, Daniel Freeman ${ }^{4,6}$, David Fowler ${ }^{9}$, Peter M. Haddad ${ }^{1,10}$, David Shiers ${ }^{1,2}$, Chris Hollis ${ }^{11}$, Jo Smith ${ }^{12}$, Ashley Liew ${ }^{3,13,14}$, Rory E. Byrne ${ }^{1,2}$, Paul French ${ }^{1,15}$, Sarah Peters ${ }^{2}$, Jemma Hudson ${ }^{7}$, Linda Davies ${ }^{16}$, Richard Emsley ${ }^{17}$, Alison Yung ${ }^{1,2,18}$, Max Birchwood ${ }^{19}$, Eleanor Longden ${ }^{1,2}$ and Anthony P. Morrison ${ }^{1,2}$

\begin{abstract}
Background: Adolescent-onset psychosis is associated with more severe symptoms and poorer outcomes than adult-onset psychosis. The National Institute for Clinical Excellence (NICE) recommend that adolescents with first episode psychosis (FEP) should be offered a combination of antipsychotic medication (APs), cognitive behavioural therapy (CBT) and family intervention (FI). The evidence for APs in treating psychosis is limited in adolescents compared to adults. Nevertheless, it indicates that APs can reduce overall symptoms in adolescents but may cause more severe side effects, including cardiovascular and metabolic effects, than in adults. CBT and FI can improve outcomes in adults, but there are no studies of psychological interventions (PI) in patients under 18 years old. Given this limited evidence base, NICE made a specific research recommendation for determining the clinical and cost effectiveness of APs versus PI versus both treatments for adolescent FEP.

Methods/design: The current study aimed to establish the feasibility and acceptability of conducting such a trial by recruiting 14-18-year-olds with a first episode of psychosis into a feasibility prospective randomised open blinded evaluation (PROBE) design, three-arm, randomised controlled trial of APs alone versus PI alone versus a combination of both treatments. We aimed to recruit 90 participants from Early Intervention and Child and Adolescent Mental Health Teams in seven UK sites. APs were prescribed by participants' usual psychiatrists. PI comprised standardised cognitive behavioural therapy and family intervention sessions.
\end{abstract}

Discussion: This is the first study to compare APs to PI in an adolescent population with FEP. Recruitment finished on 31 October 2018. The study faced difficulties with recruitment across most sites due to factors including clinician and service-user treatment preferences.

Trial registration: Current controlled trial with ISRCTN, ISRCTN80567433. Registered on 27 February 2017.

Keywords: First-episode psychosis, Cognitive behavioural therapy, Family intervention, Psychological intervention, Antipsychotic medication, Adolescent psychosis, Schizophrenia, Randomised controlled trial

\footnotetext{
* Correspondence: m.r.broome@bham.ac.uk

${ }^{3}$ Institute for Mental Health, School of Psychology, University of Birmingham,

Edgbaston, Birmingham B15 2TT, UK

${ }^{4}$ Department of Psychiatry, Medical Sciences Division, University of Oxford,

Warneford Hospital, Oxford OX3 7JX, UK

Full list of author information is available at the end of the article
}

(c) The Author(s). 2019 Open Access This article is distributed under the terms of the Creative Commons Attribution 4.0 International License (http://creativecommons.org/licenses/by/4.0/), which permits unrestricted use, distribution, and reproduction in any medium, provided you give appropriate credit to the original author(s) and the source, provide a link to the Creative Commons license, and indicate if changes were made. The Creative Commons Public Domain Dedication waiver (http://creativecommons.org/publicdomain/zero/1.0/) applies to the data made available in this article, unless otherwise stated. 


\section{Background}

Psychosis is a mental health difficulty that encompasses a range of experiences including unusual or distressing beliefs and/or hallucinations, cognitive difficulties and disorganised speech and/or behaviour. In schizophrenia, a specific psychotic disorder, positive symptoms such as hallucinations and delusions can continue episodically for years and people often experience persisting disability partly due to negative symptoms (e.g. anhedonia and motivation loss) and cognitive difficulties (e.g. memory and attention deficits) [1, 2]. Findings suggest that adolescentonset psychosis may be associated with more severe initial symptoms than adult-onset [3] and a more severe course; including greater functional impairment [4], poorer social outcomes and educational achievement [5], more days in hospital and greater readmission rates [6]. Adolescentonset psychosis and schizophrenia is associated with significant societal costs and young people with psychosis and schizophrenia accounted for $25 \%$ of adolescent psychiatric inpatient admissions in England and Wales between 1998 and 2004 [7]. Access to efficacious, evidence-based interventions for young people with psychosis is therefore vital. In this paper, we will use the term "psychosis" to represent people both with and without a diagnosis of a schizophrenia spectrum disorder. Embracing diagnostic uncertainty is essential when working with people with a first episode of psychosis (FEP), as premature diagnosis may lead to pessimism and consequently poorer outcomes [8]; additionally, clients may perceive the term "psychosis" as more acceptable than a diagnostic label such as "schizophrenia" [8].

The National Institute for Health and Care Excellence (NICE) guideline for psychosis and schizophrenia in children and young people (CG155) recommends that children and young people (CYP) should be offered oral antipsychotic medication (APs) in conjunction with psychological interventions (family intervention (FI) with individual cognitive behavioural therapy (CBT)) [9]. If a young person and their parents wish to try psychological intervention alone, CG155 suggests that the care team should advise that psychological interventions (PI) are more effective when delivered in conjunction with APs, but offer individual CBT and FI if the young person and family wish to pursue that option [9]. However, CG155 also highlights that the evidence base available for these treatments in CYP is limited and relies on evidence derived largely from studies in adults.

A systematic review of pharmacological and psychological treatments for CYP with psychosis and schizophrenia identified 19 studies of APs [10]. Meta-analysis showed small effects of APs compared to placebo for positive and negative symptoms, depression and psychosocial functioning and large effects for global symptoms. However, data quality across the studies was considered poor [10]. A more recent meta-analysis of randomised controlled trials (RCTs) confirmed the efficacy of APs in CYP with psychosis but highlighted the limitations of the evidence base, especially on safety outcomes [11]. Antipsychotics are associated with a wide range of adverse effects including cardiovascular, metabolic, hormonal and extrapyramidal adverse effects [12]. Compared to adults, CYP may be more prone to developing antipsychotic adverse effects [13]. In particular, antipsychotic-associated weight gain is greater in FEP than in multi-episode schizophrenia [14]. A study by Corell et al. [15] showed that 12 weeks of antipsychotic treatment in children and adolescents, who had less than 1 week's prior antipsychotic exposure, was associated with significant rates of obesity and new-onset categorical glucose and lipid abnormalities. For example, weight gain $>7 \%$ in the participants ranged from $56 \%$ (when participants were treated with quetiapine) to $84 \%$ (with olanzapine) [15]. To date, there are no studies evaluating the long-term safety of APs in adolescents with psychosis. Therefore, evaluating long-term cost-benefit ratios is not currently possible. For some adolescents with psychosis, the risks of APs may outweigh their benefits. Moreover, discontinuation of APs against medical advice and non-adherence are common in schizophrenia, especially in the first episode [16]. Together, these factors support the importance of assessing potential nonpharmacological treatment options for psychosis.

There are currently no trials of CBT or FI with CYP under 18 years old with psychosis [10]. There are seven low-quality studies of CBT and/or FI for psychosis in young people (aged between 15 and 24 years old). Metaanalysis of the data from these studies indicates no evidence of treatment effects on symptoms, and lowquality evidence for a combination of CBT and FI on the number of days to relapse [10]. There is better-quality evidence for the effectiveness of psychological interventions for psychosis from studies conducted in adults. Meta-analyses in adult populations suggest that a combination of CBT and APs has small but statistically significant effects on symptoms and rehospitalisation rates $[17,18]$. FI has been shown to reduce relapse rates [19], and there is a signal that CBT alone can reduce symptoms in adults who choose not to take APs, particularly in participants under 21 years old [20]. The COMPARE trial [21], which allocated people aged $16+$ years with FEP to receive either APs, CBT or a combination of both, found no differences in Positive And Negative Syndrome Scale (PANSS) scores between the AP-only and the CBT-only group, or between the combination group and the AP-only group at 12-months. However, PANSS scores were significantly lower in the combination group than in the CBT-only group at 12 months. Those in the CBT-only group reported fewer non-neurological side effects than those in the AP-only and combined groups 
[21], suggesting a potential for reduced adverse effects. A fifth of participants on the COMPARE trial (15 out of 75) were aged $16-18$ years.

In summary, the NICE guideline (CG155) group determined that the available evidence, including that from adults, was sufficiently strong to recommend a combination of APs, CBT and FI as treatments for CYP with psychosis. However, for CYP with psychosis the balance of risks and benefits of APs appears less favourable and research is needed to establish the potential for psychological treatments, alone and in combination with APs, in this population. Consequently, CG155 recommended research to determine the clinical and cost effectiveness of psychological treatment alone, compared with antipsychotic medication and compared with psychological treatment and antipsychotic medication combined [9]. Subsequently, the National Institute for Health Research (NIHR), Health Technology Assessment Programme (HTA) put out a commissioned call (HTA 51/13) to answer this research question.

The Managing Adolescent first episode Psychosis Study (MAPS) is funded by the UK NIHR HTA programme to establish whether it is feasible and acceptable to run a definitive trial examining the effectiveness of antipsychotic monotherapy versus psychological intervention (CBT plus FI) versus a combination of these treatment options in adolescents with FEP. This will answer important questions about the feasibility and design of a definitive clinical and cost effectiveness trial as recommended by NICE guideline CG155.

\section{Methods/design}

Our specific objectives were to assess (1) the proportion of eligible people that clinicians are willing to refer to the trial, and the proportions of eligible referred people that are willing to participate and to comply with their allocation; (2) the rates of adherence to follow-up assessments; (3) the characteristics of trial participants (to clarify selection criteria); (4) the feasibility and acceptability of the interventions to participants, parents and clinicians, and the appropriateness of treatment protocols; (5) the randomisation and masking procedures and (6) the validity and relevance of the measures to determine their acceptability, effectiveness and safety in a definitive trial. Furthermore, we aimed to estimate plausible ranges of sample size parameters to inform a definitive trial; finalise treatment manuals and outcome measures, and clarify training/supervision needs for delivering assessments and interventions; and assess the possibility for economies of scale, and monitor the research assistants' time use.

This study is a prospective randomised open blinded evaluation (PROBE) design, feasibility, RCT, which aimed to recruit 90 participants with FEP. As a PROBE study, persons (research assistants) that were unaware of the randomisation allocation made decisions about the scoring of outcome measures. We did not have a blinded endpoint committee, however, our independent Data Monitoring and Ethics Committee (iDMC) provided oversight of unblinding.

The randomisation ratio was 1:1:1, stratified by centre and family contact (as participants without regular family contact only received individual CBT and not FI, if randomised to the PI-only and combined groups). Randomisation at the individual level was independent and concealed, using random permuted blocks. Research assistants (RAs) performed the randomisation procedure via a study-specific website developed by the Centre for Healthcare Randomised Trials (CHaRT), the UK Clinical Research Collaboration (UKCRC) registered Clinical Trials Unit (CTU \#7) supporting the study. Aside from the trial therapists, the Trial Manager and Chief and Principal Investigators received emails informing them of randomised treatment allocation, so they could monitor adherence to allocation and provide supervision to therapists. The trial administrator was also informed via the same methods, and sent letters to participants to inform them of their allocation. Participants' care teams, including their treating psychiatrist, were also informed of allocation.

Trial RAs were blind to the participants' allocations until all outcome measures were completed for all participants. Maintaining the outcome assessor blind is crucial for effectiveness outcomes and also throughout the trial for measuring safety. The iDMC and Trial Steering committee (TSC) regularly monitored unblinding by each centre, and were able to implement corrective action if necessary. RAs and therapists received rigorous in-house training on the importance of maintaining the blind and methods to achieve this, and were required to read and sign our standard operating procedure (SOP) for retaining and managing blinding. Methods outlined in the SOP included separate offices for the therapists and RAs, protocols for answering telephones, including reminders for clinicians, participants and family members about the blind, protocols for message taking and secretarial support, separate diaries, pigeon holes and databases, using passwords and encryption of randomisation information. Any accidental unblinding was recorded. The Chief Investigator (CI) reviewed all unblinding to determine any patterns.

We recruited participants from Early Intervention in Psychosis (EIP) teams and Child and Adolescent Mental Health Services (CAMHS) in UK National Health Service (NHS) Trusts across seven sites; Greater Manchester, Lancashire, Oxfordshire and Buckinghamshire, Sussex, Northumberland, Tyne and Wear, Norfolk \& Suffolk, and Birmingham. We included 14-18-year-olds presenting with FEP who were under the care of EIP and/or CAMHS services and who were seeking help for their experiences. Participants had to be within a year of 
presentation to services with psychosis and score $4+$ on the PANSS delusions and/or hallucinations subscale(s) for a minimum of seven consecutive days, to determine their first-episode status and current symptomology. They also needed to either meet International Classification of Diseases Version 10 (ICD-10) criteria for schizophrenia, schizoaffective disorder or delusional disorder (according to the diagnosis recorded by the treating psychiatrist) or entry criteria for an EIP service. Finally, participants had to be competent to provide written, informed consent and those under 16 years old had to have a parent/guardian willing to provide additional consent to contact their child (for ethical reasons). If allocated to a PI arm, participants chose whether they wished to engage in FI. If participants did not wish to engage in FI, they were not prevented from participating in the trial and receiving CBT. No research procedures were carried out with family members, i.e. we did not collect any data on them or from them.

We excluded people who had diagnoses of a moderate/severe learning disability, ICD-10 organic psychosis, or primary alcohol/substance dependence (to ensure that our population was representative of young people with a primary problem of FEP), those who could not speak English, who scored 5+ on the conceptual disorganisation item of the PANSS (to ensure that people had capacity to engage in assessments and talking therapy), those who presented with immediate risk to themselves or others, or who had received APs or structured PI within the last 3 months prior to referral (to ensure treatment naivety). We did not exclude those with autistic spectrum disorder (ASD).

The protocol is reported in adherence with the Standard protocol items: recommendations for interventional trials (SPIRIT) guidelines; see Additional file 1.

\section{Interventions}

\section{Psychological intervention}

PI was delivered by appropriately trained therapists (clinical psychologists or other mental health professionals with relevant training in CBT for psychosis (CBTp)). Participants were offered up to 30 sessions of individual CBT over a 6month treatment window on an approximately weekly basis, and the option of up to six sessions of FI. Both CBT and FI were informed by an integrative cognitive model of psychosis [22]. This therapeutic model has been used successfully in other clinical trials in young people with psychosis, and participants within these studies provided positive qualitative interview feedback about its acceptability and usefulness [23]. Therapists took an assertive outreach approach developed from youth work principles and adopted principles from social and vocational interventions (e.g., supported education and/or employment interventions).
The overall aims of CBT were to reduce distress (particularly that arising from psychotic symptoms) and improve quality of life. CBT involved assessment and psychological formulation of people's problems and goals, to allow an individualised therapeutic approach whilst retaining standardised components and clear boundaries. CBT was collaborative, with therapist and client agreeing on problems and goals to work on, using interventions dependent on clients' individualised formulations from a range described in our published manuals [24-26]. There were a range of treatment targets including positive symptoms, comorbid problems (including anxiety, depression and substance use) and social issues such as improving relationships, developing valued social roles and maintaining functioning.

There were 4 phases to the CBT intervention: (1) engagement, assessment and formulation of problems and goals; (2) formulation-derived change strategies enabling people to work towards their individual goals; (3) development of an historical formulation, focusing on vulnerability factors leading to the development of FEP and including self-esteem work and (4) maintaining wellness and preventing relapse.

FI was grounded in a psycho-educational model of family work based on the behavioural family therapy (BFT) approach [27]. An initial session included an assessment of family understanding and appraisals of presenting difficulties, sharing the emerging psychological formulation, and agreeing a problem list and family intervention goals. Further sessions included an educational component to develop a common understanding, providing normalising and recovery-orientated information on presenting difficulties, problem-solving, communication skills training and relapse prevention planning. Families were given between-session tasks and encouraged to hold family meetings to support skills practice. Family members were also given information about local services and signposted to support for themselves, where appropriate. The approach was flexible and multi-faceted in response to needs and concerns addressing specific issues relating to adolescent-onset psychosis, including diagnostic uncertainty; dealing with emotional reactions/feelings evoked by the onset of psychosis; helping families "grieve" while encouraging a sense of agency and hope for recovery. FI was delivered in tandem with individual CBT by the same therapist. A maximum of six FI sessions were offered, spread out across the 6-month therapy window to match the pacing and content of individual CBT sessions and respond flexibly to concerns of family members as they arose. The final session was offered in collaboration with the participant's care co-ordinator to ensure continuity following trial involvement and ensure goals/strategies are shared and supported.

To ensure fidelity to the treatment protocol, therapists received initial training and weekly supervision. With 
consent, therapy sessions were audio-recorded, and a randomly selected sample of tapes (stratified by stage of therapy) rated using the Cognitive Therapy Scale - Revised (CTS-R) [28]. The tape rating process continued throughout the lifetime of the trial to guide supervision, ensure fidelity to the treatment protocol and enable corrective action to be taken if necessary. Therapists completed session records after each appointment to provide detail about session content (e.g., agenda items, change strategies used and homework tasks).

\section{Antipsychotic medication}

Participants allocated to receive APs in the monotherapy or combined arms of the trial received their prescriptions from their usual psychiatrist in their care team. The psychiatrist was asked to make prescribing decisions consistent with the NICE guideline CG155, which recommends that clinicians should jointly decide the choice of antipsychotic medication with the young person and their parents/carers. The decision about medication should include discussion about the possible benefits and side effects of each drug, and provision of ageappropriate information [9]. AP prescribing should also be accompanied by physical health monitoring [9].

Psychiatrists were encouraged to initiate AP treatment as soon as possible following randomisation into the study, and to maintain AP treatment preferably for 26 weeks, but for 12 weeks minimum. The decision about the type and dose of AP was made by the psychiatrist as per their usual practice, i.e. we did not ask them to choose from a pre-specified list of antipsychotic medications. Psychiatrists were free to change dose and type of $\mathrm{AP}$ in response to efficacy and adverse events. A member of the research team who was not blind to allocation screened participants' medical records to collect details about AP prescription. Additionally, self-report data were provided by participants either via our web-based platform or if preferred, in paper format that was returned to a research team member who was not blind to allocation. Psychiatrists on the study were available for consultation if participants' CAMHS or EIP psychiatrists wished to discuss AP prescription.

We did not restrict the treatment options offered by the care team as it would have been unethical to do so. Additionally, participants were eligible to receive mental health medications other than APs, and psychological therapies other than and including CBTp or FI, throughout the course of the trial. A member of the research team who was not blind to allocation screened participants' medical records for information on concomitant therapies received. In addition, self-report data on concomitant therapies were provided by participants using the method described above. Data from participants' medical records and self-report data provided important feasibility information about participants' adherence to their randomised treatment allocation.

\section{Outcomes}

We do not have a single primary outcome, as this is not meaningful for a feasibility study. Our key outcomes to inform a definitive trial are rates of referral, recruitment, therapy attendance, adherence to medication, and completion of follow-up appointments and questionnaires. The acceptability of treatment will be determined by measuring discontinuation rates and through data collected within a nested qualitative study (see below). We have specific red/amber/green progression criteria that have been agreed with our iDMC, TSC and funder. Green would mean progression to a full trial is possible without the need for any substantial changes to the design or the way it was delivered; amber would mean we may need more resource to recruit, and ways of improving retention and compliance; and red would mean there is substantial doubt that the definitive study is feasible at an affordable cost. These progression criteria will be reviewed by the iDMC and TSC at the end of the trial and will inform a recommendation for a definitive trial. The progression criteria to a future definitive trial are described below:

1. Recruitment $\geq 80 \%$ of planned (green), recruitment within $79-60 \%$ of planned (amber), recruitment $<60 \%$ of planned (red).

2. Retention of participants within the study with baseline and outcome assessments at primary end point ( 6 months, end of treatment) $\geq 80 \%$ of primary outcome completed (green), 79-60\% of primary outcome completed (amber), $<60 \%$ of primary outcome completed (red).

3. Satisfactory delivery of adherent therapy to $\geq 80 \%$ of groups receiving PI (green), 79-60\% of groups receiving PI (amber), <60\% of groups receiving PI (red). Satisfactory delivery of adherent therapy is operationalised as attending 6 or more sessions of CBT.

4. Satisfactory delivery of antipsychotic medication to $\geq 80 \%$ of groups receiving AP (green), 79-60\% of groups receiving AP (amber), $<60 \%$ of groups receiving AP (red). Satisfactory delivery of antipsychotic medication is operationalised as any exposure of AP for six consecutive weeks (this would include a dose below British National Formulary (BNF) lower limits given this is a frequent clinical practice for people of this age, and APs are licensed for adults).

We have a number of secondary outcomes, which were collected from participants via self-report and interview measures at baseline and follow-up 
appointments (see Fig. 1 for schedule of enrolment, interventions and assessments). This is to assess the acceptability and usefulness of the measures for inclusion in a definitive trial, rather than to measure the relative safety/efficacy of the interventions. Our provisional choice of primary outcome measure for a definitive trial is total PANSS score, which would ensure comparability to other antipsychotic and psychological therapy trials; however, a final decision on a primary outcome will need to be informed by data from this trial, associated qualitative studies and stakeholder opinion. The PANSS is a 30 -item rating scale for psychopathologic assessment of adults with a diagnosis of schizophrenia [29]. It is a frequently used outcome measure in psychosis research. We assessed social and educational/occupational functioning with the First Episode Social Functioning Scale (FESFS) [30], a questionnaire developed with people presenting with FEP that has good reliability, validity and sensitivity to change. We measured subjective recovery using the 15-item version of the Questionnaire about the Process of Recovery (QPR) [31], a questionnaire developed in collaboration with people with psychosis. We used the Specific Psychotic Experiences Questionnaire (SPEQ) [32] to assess paranoia, hallucinations, cognitive disorganisation, grandiosity and anhedonia.

To measure common comorbidities, we used (1) the Hospital Anxiety and Depression Scale (HADS) [33], which has shown to be reliable and valid in measuring anxiety and depression symptoms over the past 7 days; (2) the Alcohol Use Disorder Identification Test (AUDIT) [34] and (3) the Drug Abuse Screening Test (DAST) [35]. The latter two measures are statistically predictive of the respective substance misuse disorders, using the Structured Clinical Interview for the Diagnostic and Statistical Manual version 5 (DSM-IV) (SCIDIV) [36]. We also measured diagnostic symptoms for autism spectrum conditions at baseline using the NICErecommended 10-item version of the Autism Spectrum Quotient (AQ-10) [37]. We collected data on adverse effects of medication and trial participation (described

\begin{tabular}{|c|c|c|c|c|c|c|c|c|c|c|c|c|c|c|c|c|}
\hline & Enrolment & & & & & ost & loce: & on 1 & onth & & & & & & & \\
\hline TIMEPOINT & 0 & 1 & 2 & 3 & 4 & 5 & 6 & 7 & 8 & 9 & 10 & 11 & 12 & 3MFU & 6MFU & 12MFU \\
\hline Eligibility screen & $X$ & & & & & & & & & & & & & & & \\
\hline Informed consent & $X$ & & & & & & & & & & & & & $x$ & $\mathrm{x}$ & $x$ \\
\hline Baseline assessment & $X$ & & & & & & & & & & & & & & & \\
\hline Allocation & $\mathrm{X}$ & & & & & & & & & & & & & & & \\
\hline INTERVENTIONS: & & & & & & & & & & & & & & $\mathrm{x}$ & $x$ & $\mathrm{x}$ \\
\hline$[P I]$ & & & & & & & & & & & & & & $\mathrm{x}$ & $x$ & $\mathrm{x}$ \\
\hline [APs] & & & & & & & & & & & & & & $x$ & $\mathrm{X}$ & $\mathrm{x}$ \\
\hline$[P I+A P s]$ & & & & & & & & & & & & & & $x$ & $x$ & $x$ \\
\hline ASSESSMENTS: & & & & & & & & & & & & & & $x$ & $\mathrm{x}$ & $\mathrm{x}$ \\
\hline $\begin{array}{r}\text { ANNSERS, AUDIT, } \\
\text { DAST, Demographics, } \\
\text { EQ-5D + EPQ, FESFS, } \\
\text { HADS, PANSS, } \\
\text { Physical health } \\
\text { checks, QPR }\end{array}$ & $x$ & & & & & & & & & & & & & $x$ & $x$ & $\mathrm{x}$ \\
\hline $\begin{array}{r}A Q-10, \text { DUP } \\
\text { questionnaire }\end{array}$ & $\mathrm{x}$ & & & & & & & & & & & & & & & \\
\hline $\begin{array}{l}\text { Concomitant therapies } \\
\text { form }\end{array}$ & & & & & & & & & & & & & & $\mathrm{x}$ & $x$ & $\mathrm{x}$ \\
\hline $\begin{array}{l}\text { Experiences of trial } \\
\text { participation form }\end{array}$ & & & & & & & & & & & & & & & $x^{*}$ & $x$ \\
\hline
\end{tabular}

Fig. 1 Schedule of enrolment, interventions and assessments. APS, antipsychotic medication; ANNSERS, Antipsychotic Non-Neurological Side Effects Scale; AQ-10, Autism Spectrum Quotient 10-item version; DAST, Drug Abuse Screener Test; DUP, duration of untreated psychosis; EPQ, Economic Patient Questionnaire; EQ-5D, EuroQol five dimension scale; FESFS, First Episode Social Functioning Scale; HADS, Hospital Anxiety and Depression Scale; PANSS, Positive And Negative Syndromes Scale; PI, psychological intervention; QPR, Questionnaire about the Process of Recovery; 3MFU, 6MFU, 12MFU 3-month, 6-month, 12-month follow up. *Only for participants who were randomised after the first 10 months of the trial and thus were not offered a $12 \mathrm{MFU}$ 
further under "Safety Monitoring"). We collected basic health economics data about services used by participants, using an economic patient questionnaire adapted from previous studies conducted by the authors [38, 39] and the EuroQol five dimension, five level scale (EQ-5D$5 \mathrm{~L})$ health status questionnaire [40]. This information will inform the design of the economic component of a definitive trial.

We designed a variable-length follow-up period, whereby participants recruited in the first 10 months were offered assessments at 3, 6 and 12 months and those recruited thereafter were only offered assessments up to the end of treatment ( 6 months, the proposed timing for the primary outcome). RAs collected all outcome measures at baseline and at the 3-month, 6-month and 12-month follow-up assessments. RAs underwent inhouse training in administering all the measures to establish sufficient inter-rater reliability, including watching and scoring role-play and videos. These training sessions occurred prior to RAs delivering assessments independently and on several occasions throughout the course of the trial, to prevent "rater drift". Measures were administered in a specific order agreed by the trial manager and $\mathrm{CI}$ to prioritise the most important (beginning with the PANSS).

To promote retention to the trial and reduce participant burden, RAs worked in a person-centred manner and gave participants control where possible over the location and time of appointments, offered breaks and multiple visits to complete measures, and gave the option of skipping measures if participants found them difficult to complete. Participants were compensated $£ 10$ per assessment and RAs contacted participants between follow-up appointments with a "check-in" phone call and a $£ 5$ shopping voucher. These processes are consistent with systematic review evidence for increasing retention in clinical trials [41]. RAs received weekly trial management supervision to enable monitoring of follow-up rate retention and to proactively problem-solve issues with completing assessments.

\section{Safety monitoring and reporting}

We took a rigorous approach to recording and reporting serious adverse events. We recorded all serious adverse events (SAEs) at each point of contact with participants after randomisation. Our definition of an SAE was informed by the standard Health Research Authority definition and our trial protocol, which included all deaths, life-threatening incidents including suicide attempts, serious violent incidents, hospital admissions including admissions to secure units (we record whether these are voluntary or involuntary) and physical health units, prolongation of hospitalisation, any events resulting in persistent or significant disability or incapacity, any event consisting of a congenital abnormality or birth defect and formal complaints about treatment.

As noted, we scrutinised any instances of participants being admitted to psychiatric hospitals throughout the trial. The therapists or RAs were likely to have become aware of adverse events or admissions to psychiatric hospitals; however, we also screened each participant's medical notes to assess for adverse events. All SAEs were monitored by the iDMC and TSC. All related and unexpected SAEs were reported to the National Research Ethics Committee (NREC) and the participant's NHS Trust. We measured potential adverse effects associated with trial participation at the participant's final follow-up assessment (i.e. 6 months or 12 months, dependent on when they were randomised into the trial), using a measure developed in our HTA-funded FOCUS trial [42]. In addition, if a participant in the monotherapy arm experienced a substantial deterioration in their mental health throughout the study, they were offered a switch to the combined treatment arm. This would be offered if the participant's mental state had significantly deteriorated from baseline at the 3-month follow-up appointment (operationalised by an increase of $12.5 \%$ or more in rescaled PANSS scores) or if they were admitted involuntarily to hospital at any point in the trial.

We measured adverse effects of medication; firstly by interviewing participants using the Antipsychotic NonNeurological Side Effects Scale (ANNSERS) [43], and secondly by measuring weight, height, waist circumference and blood pressure. In addition, a blood sample was taken for assessment of fasting plasma glucose (FPG), glycated haemoglobin (HbA1C), serum prolactin levels, and lipids (total cholesterol, low-density lipoproteins (LDL), high-density lipoproteins (HDL) and triglycerides). ANNSERS, physical examinations and blood tests were conducted at baseline and at the 3-month, 6month and 12-month assessment. The results of blood tests were sent to participants' psychiatrists (or other responsible clinician) at each time point. Finally, during screening of participants' medical records for SAEs as described above, we recorded any adverse events related to antipsychotic medication (i.e., side effects) that were reported in participants' notes.

\section{Consent}

RAs were responsible for consenting participants into the trial under the supervision of the Chief and Principal Investigators. In addition to receiving Good Clinical Practice training, RAs completed the online module "Informed Consent in Paediatric Research" offered by the NIHR. Parents/legal guardians and potential participants had at least $24 \mathrm{~h}$ to read the Research Ethics Committee (REC)-approved participant information sheet, before meeting with an RA for the informed consent visit. If 
the young person was under 16 years old, RAs were required to obtain written informed consent from their parent/legal guardian to contact the young person prior to taking written informed consent to participate from the young person. The RAs followed the same procedure as above to ensure parents/guardians were fully informed and understood the study information, and if satisfied, the RA requested the parent to sign an assent form allowing the RA to contact their child about the study. The RA could then arrange to meet with the young person to sign their own consent form. Ongoing consent was confirmed at each research assessment and documented in the participant's research notes.

\section{Trial oversight}

The Sponsor of the trial is Greater Manchester Mental Health NHS Foundation Trust. The Sponsor was responsible for auditing the conduct of the trial. Independent oversight of the trial was provided by the iDMC and TSC. The iDMC was composed of an independent chairperson, statistician, clinician and service user. The role of the iDMC was to monitor recruitment of study participants, ethical issues of consent, quality of data (including missing data), the incidence of adverse events, unblinding and withdrawals and any other factors that might have compromised the progress and satisfactory completion of the trial. The iDMC met on a 6-monthly basis. A copy of the iDMC charter was retained by the Trial Manager in the site file.

The TSC was composed of an independent chairperson, statistician, clinician and service user along with nonindependent members; the $\mathrm{CI}$, trial manager, a representative of the funder (NIHR HTA) and a representative of the Sponsor. The TSC met every 6 months to monitor and supervise progress, and consider reports and recommendations. Meetings occurred approximately $2-4$ weeks after the iDMC so the iDMC report could inform the TSC meeting. Prior to each iDMC and TSC meeting, the independent members were required to declare any conflicts of interest. The outcome of each iDMC and TSC meeting was reported to the funder via the minutes of the meeting. The trial management group, which comprised grant applicants and other local investigators met on an approximately monthly basis to provide management oversight of recruitment, attrition, adverse events, blind breaks, withdrawals and data management. Following the addition of three more sites in May and June 2018, individual site management meetings were organised on a monthly basis to ensure oversight of site-specific issues.

\section{Data management}

All of the information collected about the participants is strictly confidential. All participants' personal identifiable data (PID) are kept securely in databases, on secure NHS computer drives that are only accessible to the research team and are protected by a password known only by study staff. All RAs' computers are also individually passwordprotected. Paper copies of research data (i.e. assessment packs) are anonymised with a trial identification (ID) number and kept in a locked, filing cabinet separate to the storage of PID (such as referral forms and letters about the participant). Participants were made aware that although their data are strictly confidential and not shared outside of the research team, confidentiality could be broken in cases where participants were deemed to pose a risk to themselves or to others.

All research data entered on the secure web-based platform created by $\mathrm{CHaRT}$ were completely anonymised. To ensure the accuracy of the data entry for the proposed primary outcome measure (PANSS), RAs checked entries for every participant by comparing PANSS scores in the paper assessment files against the scores entered into the $\mathrm{CHaRT}$ platform. Data were checked once all possible assessments for each time point were completed. An error rate of $2 \%$ or less was deemed acceptable. If the error rate had been above $2 \%$ the trial statistician and methodologist would have advised on further data checking, although this was not necessary due to the accuracy of the data entry.

The final trial dataset is managed and held by our CTU, $\mathrm{CHaRT}$. Requests for access to the dataset will be considered in the first instance by the $\mathrm{CI}$ and then the CTU.

\section{Sample size}

We proposed a sample size of 90 participants across all sites (30 per treatment arm). The target sample size is sufficient to attain reliable sample size estimates [44], gain feasibility information about trial proceures and facilitate a power calucalation. No power calculation was performed for this study as the focus of feasibility study analysis is not hypothesis testing [45]. We will estimate 95\% confidence intervals to indicate likely intervention effects for a definitive trial.

\section{Recruitment}

We had a team of highly motivated research staff who were experienced in recruiting participants into clinical trials, working across carefully selected NHS sites with which we have successfully collaborated on previous trials. Many of our sites had established relationships with EIP and CAMHS teams due to having research staff embedded within the teams, and/or due to recruitment efforts in previous trials. All sites covered extensive geographical areas. We used a joint-working approach between research and clinical teams in each site to build positive relationships with clinical staff, identify all potential cases and encourage clinical staff to discuss the trial with service users to enhance the referral rate. This included regular contact between the RAs and the 
clinical teams. Supervision and team meetings allowed RAs to provide feedback on teams they were struggling to contact so that the team could collectively problemsolve ways of moving forward.

To ensure fidelity to the allocated treatment, we endeavoured to work closely with EIP and CAMHS psychiatrists. The exclusion criterion of AP treatment naivety meant that engaging those who prescribe to CYP with psychosis was an important aspect to recruitment. The trial design required the participant's usual psychiatrist (within their care team) to prescribe an AP as soon as possible after randomisation, if the participant was allocated to the AP monotherapy or combined treatment arms. To engage EIP and CAMHS psychiatrists we ran continuing professional development (CPD)/training events, and the CI directly contacted psychiatrists to ask for their support. Additionally, NIHR-funded clinical studies officers (CSOs) within many sites had existing relationships with teams and helped promote the study, identified research-motivated staff and where possible reviewed team caseloads for potential MAPS candidates.

MAPS began recruiting in April 2017 as a four-site trial with a 15-month recruitment window (projecting 1.5 randomisations per month per site). The actual versus target site-recruitment varied considerably between sites. Barriers to recruitment were identified across all sites. In some instances, there was a strong treatment preference expressed by clinicians, young people and/or parents, which influenced referral to the trial and/or young people agreeing to take part. The incidence rates of young people either being referred to, or accepted into EIP teams were relatively low across all sites, although numbers did vary by site. We also found variation in the inclusion/exclusion criteria of EIP services both across and within sites, particularly in terms of applying diagnostic uncertainty to people's experiences. For example, some teams did not accept people whose psychotic experiences were judged a product of mood, trauma or personality-related difficulties, or where the duration of untreated psychosis reached a particular threshold. As a result, in some instances this led to a discrepancy between young people's eligibility for MAPS and for an EIP service. As AP prescription was made by the participant's usual psychiatrist, entry into the trial was dependent on the young person's psychiatrist agreeing that randomisation to either or both MAPS treatments would be appropriate. The discrepancy between MAPS inclusion criteria and individual EIP inclusion criteria therefore further limited the already small number of young people who were potentially eligible for the trial. Additionally, due to the necessity of psychiatrists agreeing to young people entering the trial (including not prescribing APs for them prior to trial entry as described above), engaging psychiatrists with positive views of the trial embedded within EIP/CAMHS teams was crucial for recruitment. This was not possible across all services due to clinicians' individual views and practices in relation to the treatment of FEP in young people. Another factor that proved challenging to recruitment was the relatively large proportion of young people who had already been prescribed APs prior to initial contact with EIP/ CAMHS services (for example, via care pathways involving crisis/home treatment teams or inpatient services).

The most successfully recruiting MAPS site engaged psychiatrists embedded within EIP who regularly conducted service assessments, and could therefore provide an opinion on whether there was clinical equipoise for a young person early into their accessing services (before prescription of APs is made). In order to evaluate the feasibility of replicating this model approach to recruitment we added three new sites (Birmingham, Norfolk \& Suffolk and Northumberland, Tyne and Wear) in May 2018, and extended our recruitment window by four months to 31 October 2018.

\section{Data analysis}

The main aims of the feasibility trial will be delivered both via the continued monitoring of descriptive data and the analysis of data following the last follow-up assessment. Analysis is ongoing and began after full recruitment and follow up (i.e. there were no interim analyses for efficacy, although the iDMC monitored trial progress and any safety issues on a regular basis).

The main analyses are based on an intention-to-treat approach, using all randomised participants. Since safety and unwanted effects should be analysed on the basis of the most accurate information, these analyses are based on treatment received rather than as-randomised. Treatment received for the analysis of safety and unwanted effects is defined as any dose of an antipsychotic prescribed by the participant's responsible psychiatrist and any dose of CBTp or FI from a trial therapist. We will use descriptive statistics to summarise the key indicators of success of the trial, including participant recruitment; checks for absence of selective recruitment of participants; baseline balance and participant flow. We will report data in line with the consolidated standards of reporting trials (CONSORT) 2010 extension statement for pilot and feasibility trials [46]. Important summary statistics will be the number of participants referred through case managers and mental health staff, number of referrals found to be eligible and number of consenting individuals and recruited individuals to each arm. Numbers for discontinuation from the allocated interventions, withdrawal of consent and failure to provide follow-up outcome data will also be generated. We will also report the proportion of participants who received their allocated intervention compared to the proportion who did not, and the proportion of 
participants offered a move to the combined therapy arm due to deterioration.

We will report our feasibility results (recruitment, retention, adherence) overall, in order to inform decisions about the viability of a future definitive trial. However, we will also report our descriptive results and $95 \%$ confidence intervals on outcome measures by group. We will describe actual treatment received and treatment compliance (to account for departures from the randomised interventions). We will also report descriptive statistics for the components of psychological intervention received, including number of sessions and milestones achieved.

To inform a phase III trial we will conduct the following analysis to ensure the data conform to the assumptions of the tests that will be conducted at that stage: measures proposed as the primary (PANSS) and secondary outcomes (QPR) for the phase III study will be analysed by analysis of repeated measures using a mixed effects model to account for the discrete timing of the follow-up assessments. The presentation of the analysis will focus on point estimates and associated 95\% confidence intervals rather than statistical significance ( $p$ values). Further analysis will test the correlation of each measure across all time points and the variation within the proposed outcome measure (mean and standard deviation) to inform a definitive sample size calculation for a phase III trial. No formal analysis will be performed to account for missing data as MAPS is a feasibility study.

We will measure within trial and also explore the literature on the possible effects of clustering by therapist and site. We anticipate that the chance of clustering in relation to drug outcomes will be negligible, given that we are expecting prescribing to follow NICE (CG155) guidance, although we will measure this in case of significant differences in prescribing practices between sites. We will adjust for site in analyses (therapist will be nested within site) and examine intraclass correlation coefficients to inform a plan for managing any such impact on design and analysis of a definitive trial.

All statistical analyses are pre-specified in a comprehensive statistical analysis plan (SAP) authored by the study statistician and agreed with the iDMC and TSC.

\section{Intervention and trial acceptability: qualitative interviews} A nested qualitative study aimed to identify key themes associated with the acceptability of the trial and interventions amongst CYP, family members/carers and clinicians. Individual semi-structured interviews explored participants' subjective experiences of recruitment, random allocation and receiving interventions, including views of adverse effects and benefits; sought to identify barriers and solutions to participation; and ultimately aimed to identify themes relating to all of these issues. This will inform the design of a definitive trial and help further refine intervention, recruitment and retention procedures. We sought a maximum variance sample on key variables among participants (gender, age, ethnicity, site, engagement with interventions). The CYP interviews were conducted with participants from all treatment arms within the trial after 6 month assessments; this allowed us to explore people's experiences of receiving the treatments and of participating in the trial. Based on our previous work we expected thematic saturation would be achieved within 15-20 CYP interviews [47], 15-20 family members/carers [48] and 15-20 clinicians. All interviews were audio-recorded and transcribed verbatim at which point any identifying information (names and places) was removed. Data are currently being thematically analysed [49]. This method results in a rich and accessible account of qualitative data through the researcher making sense of the data and reporting themes that emerge [49]. Data were coded systematically and iteratively, and were organised within Nvivo qualitative data analysis Software version 11 [50].

\section{Dissemination}

The results from this study will be published in a peerreviewed journal for dissemination amongst researchers and clinicians. We will follow the International Committee of Medical Journal Editors (ICMJE) recommendations for authorship and review these for each individual publication. The results will also be disseminated to participants, if they agree to this. Participants leaving the trial were asked whether they would like the results of the study and their preferred method for receiving them (e.g. post, telephone, email). The results will also be disseminated amongst the healthcare professionals and teams that have helped support recruitment for the trial.

\section{Discussion}

Our trial is the first of its kind, as we are not aware of any other feasibility trials that have recruited young people with FEP into a three-arm RCT of AP monotherapy, PI monotherapy or a combined treatment. Our trial has a number of strengths including using methods to minimise selection bias, such as generation of randomised permuted blocks via a computer system and centralisation of allocation via a web-based platform. We adhered to detailed operational procedures to minimise detection bias as outlined in our SOP for maintaining the blind. We have a low risk of selective reporting as we have published our SAP on the CTU website following agreement by our iDMC and TSC. We used a rigorous approach to recording and reporting SAEs, including reports from trial staff and medical record screening for details of SAEs, as specified in our iDMCapproved SOP for SAEs. Moreover, we go beyond UK Health Research Authority (HRA) requirements for 
review of SAEs and in addition to review by the $\mathrm{CI}$, the iDMC chairperson provided an independent review of whether each SAE is trial-related or not. In addition, our trial included a bespoke measure of potentially unwanted effects of trial participation.

In summary, APs and psychological interventions (PI), specifically, family intervention and cognitive behaviour therapy are recommended treatments for FEP in adolescents but evidence about relative efficacy/acceptability is limited. If PI were non-inferior to APs, within an acceptable margin, this could be a major advance in treating a vulnerable group with high sensitivity to APs. The MAPS trial provides crucial data on the feasibility and acceptability of conducting a phase III trial of these treatments and the conditions that would be required to conduct such a trial.

\section{Trial status}

Recruitment began on 1 April 2017 and ended on 31 October 2018 with a total of 61 participants randomised into the trial across six of the seven sites: 25 in Oxfordshire and Buckinghamshire, 21 in Greater Manchester, 9 in Lancashire, 4 in Sussex, 1 in Northumberland, Tyne and Wear and 1 in Birmingham. This represents $67.8 \%$ of the original target of 90 participants. All PI sessions were finalised across all sites by 30 April 2019. All follow-up assessments were completed and data entered by 2 May 2019 . Qualitative interview recruitment commenced on 1 September 2017 and finished on 22 January 2019. Statistical and qualitative analyses are currently ongoing. This paper represents version 5 (17 July 2018) of the MAPS protocol. All Principal Investigators, the REC and HTA were informed of protocol modifications.

\section{Additional file}

Additional file 1: SPIRIT 2013 checklist: recommended items to address in a clinical trial protocol and related documents. (DOC $121 \mathrm{~kb}$ )

\footnotetext{
Abbreviations

ANNSERS: Antipsychotic Non-Neurological Side Effects Scale; APs: Antipsychotic medication; AQ-10: Autism Spectrum Quotient 10-item version; ASD: Autistic spectrum disorder; AUDIT: Alcohol Use Disorders Identification Test; BFT: Behavioural family therapy; BNF: British National Formulary; CAMHS: Child and Adolescent Mental Health Services; CBT: Cognitive behavioural therapy; CBTp: Cognitive behavioural therapy for psychosis; CG155: Guideline for psychosis and schizophrenia in children and young people; CHaRT: Centre for Healthcare Randomised Trials; Cl: Chief Investigator; CONSORT: Consolidated standards of reporting trials; CPD: Continuing professional development; CSO: Clinical Studies Officer; CTS-R: The revised Cognitive Therapy Scale; CTU: Clinical Trials Unit; CYP: Children and young people; DAST: Drug Abuse Screener Test; DSM-IV: Diagnostic and Statistical Manual version 5; EIP: Early intervention in psychosis; EME: Efficacy and Mechanism Evaluation; EPQ: Economic Patient Questionnaire; EQ-5D: EuroQol five dimension scale; FEP: First episode of psychosis; FESFS: First Episode Social Functioning Scale; Fl: Family intervention; FPG: Fasting samples of plasma glucose; HADS: Hospital Anxiety and Depression Scale; HbA1c: Glycated haemoglobin; HDL: Highdensity lipoproteins; HRA: Health Research Authority; HTA: Health Technology Assessment; ICD-10: International Classification of Diseases Version 10;
}

ICMJE: International Committee of Medical Journal Editors; ID: Identification; iDMC: Independent Data Monitoring Committee; iTSC: Independent Trial Steering Committee; LDL: Low-density lipoproteins; MAPS: Managing Adolescent Psychosis Study; NCCMH: National Collaborating Centre for Mental Health; NHS: National Health Service; NICE: National Institute for Health and Care Excellence; NIHR: National Institute for Health Research; NREC: National Research Ethics Committee; PANSS: Positive And Negative Syndromes Scale; PI: Psychological intervention; PID: Patient identifiable data; PROBE: Prospective randomised open blinded evaluation; QPR: Questionnaire about the Process of Recovery; RA: Research Assistant; RCT: Randomised controlled Trial; REC: Research Ethics Committee; SAE: Serious adverse event; SAP: Statistical analysis plan; SCID-IV: Structured Clinical Interview for DSM-IV; SOP: Standard operating procedure; SPEQ: Specific Psychotic Experiences Questionnaire; SPIRIT: Standard protocol items: recommendations for interventional trials; TM: Trial Manager; TMM: Trial Management Group; TSC: Trial Steering committee; UKCRC: UK Clinical Research Collaboration

\section{Acknowledgements}

Thank you to all the participants, family members/carers who agreed to take part in the trial and clinicians who have supported participant enrolment into the trial. This study was supported by the National Institute for Health Research (NIHR), the NIHR MindTech MedTech Co-operative, the NIHR Nottingham Biomedical Research Centre and the Clinical Research Network-Mental Health. MBi is part funded by NIHR CLAHRC West Midlands. We are grateful to the Psychosis Research Unit (PRU) Service User Reference Group (SURG) for their consultation regarding the design of the study and contribution to the developments of study-related materials. We are grateful to our Independent Trial Steering Committee and Independent Data Monitoring Committee for providing oversight of the trial.

\section{Authors' contributions}

MP made substantial contribution to the development of the trial protocol and to the overall management of the trial and data management, and wrote the first draft of the manuscript. AL, AY, CH, DFo, DFr, DS, EL, LD, PF, $\mathrm{SP}, \mathrm{MBi}, \mathrm{MBr}$ and $\mathrm{REB}$ contributed to the application for funding, made substantial contribution to the design of the trial and protocol and critically read the manuscript. EJ contributed to the writing of the manuscript. GM made substantial contribution to the design of the trial, the protocol and the statistical analysis plan and critically read the manuscript. JN, RE contributed to the application for funding, made substantial contribution to the design of the trial, the protocol and the statistical analysis plan and critically read the manuscript. PH made substantial contribution to the design of the trial and protocol and critically read the manuscript. JS made a substantial contribution to the family intervention component of the protocol, provided $\mathrm{Fl}$ supervision to therapists on the trial and contributed to the writing of the manuscript. JH made substantial contribution to the statistical analysis plan and critically read the manuscript. APM planned the study, contributed to the application for funding, made substantial contribution to the design of the trial protocol and the statistical analysis plan, managed the stud, and critically read the manuscript. All authors read and approved the final manuscript.

\section{Funding}

MAPS was funded by the NIHR HTA programme (project number 15/31/04) and the final report will be published in Health Technology Assessment. Visit the HTA programme website for further project information.

The Sponsor is Greater Manchester Mental Health NHS Foundation Trust, email R\&D@gmmh.nhs.uk.

The views and opinions expressed therein are those of the authors and do not necessarily reflect those of the HTA programme, NIHR, NHS or the Department of Health.

\section{Availability of data and materials \\ Not applicable.}

\section{Ethics approval and consent to participate}

MAPS was approved by the North West - Greater Manchester East REC on 06.02.2017 (reference 16/NW/0893). All participants provided written informed consent to participate in MAPS. For participants under 16 years old, parents/ legal guardians also gave written informed consent for the trial team to contact their child before the participant could provide their consent. 


\section{Consent for publication}

Not applicable.

\section{Competing interests}

We have the following conflict of interests to declare. All authors have conducted funded research into treatments for psychosis. APM has received royalties for CBT manuals and practises CBT in the NHS. DS is expert advisor to the NICE centre for guidelines and a member of the current NICE guideline development group for Rehabilitation in adults with complex psychosis and related severe mental health conditions; Board member of the National Collaborating Centre for Mental Health (NCCMH); views are personal and not those of NICE or NCCMH. PF has received royalties for CBT manuals, is a member of the NIHR HTA Mental Health Prioritisation Panel and Joint National Clinical Advisor to the National Audit of Psychosis. PMH has received fees for lecturing and/or consultancy work from Janssen, Lundbeck, NewBridge Pharmaceuticals, Otsuka and Sunovion plus conference support from Janssen, Lundbeck, NewBridge Pharmaceuticals and Sunovion. JN declares membership of the following NIHR boards: CPR decision making committee; HTA Commissioning Board; HTA Commissioning Sub-Board (EOI); HTA Funding Boards Policy Group; HTA General Board; HTA Post-Board funding teleconference; NIHR CTU Standing Advisory Committee; NIHR HTA \& EME (Efficacy and Mechanism Evaluation) Editorial Board; Pre-exposure Prophylaxis Impact Review Panel. JS is Joint

National Clinical Advisor to the National Audit of Psychosis and has received fees for lecturing and/or consultancy work from Janssen and Otsuka and book royalties from Wiley-Blackwell publishers. REB has received fees for teaching related to CBTp, and is an invited expert reviewer for the NIHR Health Services and Delivery Programme.

\section{Author details}

${ }^{1}$ The Psychosis Research Unit, Department of Psychology, Greater Manchester Mental Health NHS Foundation Trust, Prestwich M25 3BL, UK ${ }^{2}$ Division of Psychology and Mental Health, University of Manchester, Zochonis Building, Manchester M13 9PL, UK. ${ }^{3}$ Institute for Mental Health, School of Psychology, University of Birmingham, Edgbaston, Birmingham B15 $2 \pi$, UK. ${ }^{4}$ Department of Psychiatry, Medical Sciences Division, University of Oxford, Warneford Hospital, Oxford OX3 7JX, UK. ${ }^{5}$ Centre for Human Brain Health, School of Psychology, University of Birmingham, Edgbaston, Birmingham B15 2TT, UK. ${ }^{6}$ Oxford Health NHS Foundation Trust, Warneford Hospital, Oxford OX4 7JX, UK. ${ }^{7}$ Centre for Healthcare Randomised Trials, Health Services Research Unit, University of Aberdeen, 3rd Floor Health Sciences Building, Aberdeen AB25 2ZD, UK. ${ }^{8}$ Edinburgh Clinical Trials Unit, Centre for Population Health Sciences, Usher Institute, Nine Edinburgh BioQuarter, 9 Little France Road, Edinburgh EH16 4UX, UK. ${ }^{9}$ School of Psychology, Pevensey Building, University of Sussex, Falmer BN1 9QH, UK. ${ }^{10}$ Department of Psychiatry, Hamad Medical Corporation, PO Box 3050, Doha, Qatar. ${ }^{11}$ NIHR MindTech MedTech Co-operative, Division of Psychiatry and Applied Psychology, Institute of Mental Health, University of Nottingham, Innovation Park, Triumph Road, Nottingham NG7 2TU, UK. ${ }^{12}$ School of Allied Health and Community, Bredon Building, University of Worcester, Worcester WR2 6AJ, UK. ${ }^{13}$ Centre for Educational Development, Appraisal and Research, University of Warwick, Coventry CV4 7AL, UK. ${ }^{14}$ Forward Thinking Birmingham, Birmingham Women's and Children's NHS Foundation Trust, Finch Road, Lozells B19 1HS, UK. ${ }^{15}$ Institute of Psychology, Health and Society, University of Liverpool, Waterhouse Building, Liverpool L69 3BX, UK. ${ }^{16}$ Division of Population Health, Health Services Research \& Primary Care, University of Manchester, Jean MacFarlane Building, Manchester M13 9PL, UK. ${ }^{17}$ Department of Biostatistics \& Health Informatics, Institute of Psychiatry, Psychology \& Neuroscience, King's College London, Denmark Hill, London SE5 8AZ, UK. ${ }^{18}$ Centre for Youth Mental Health, Faculty of Medicine, University of Melbourne, 35 Poplar Rd, Parkville, Melbourne, VIC 3052, Australia. ${ }^{19}$ Warwick Medical School-Mental Health and Wellbeing, University of Warwick, Coventry CV4 7AL, UK.

\section{Received: 17 October 2018 Accepted: 10 June 2019}

Published online: 04 July 2019

\section{References}

1. Crismon L, Argo TR, Buckley PF. Schizophrenia. In: DiPiro JT, Talbert RL, Yee GC, et al., editors. Pharmacotherapy: a pathophysiologic approach. 9th ed. New York: McGraw-Hill; 2014. p. 1019-46.
2. Lavretsky $\mathrm{H}$. History of schizophrenia as a psychiatric disorder. In: Mueser KT, Jeste DV, editors. The clinical handbook of schizophrenia. New York: The Guildford Press; 2008. p. 3-13.

3. Langeveld J, Joa I, Friis S, ten Velden Hegelstad W, Melle I, Johannessen $\mathrm{JO}$, et al. A comparison of adolescent- and adult-onset first-episode, non-affective psychosis: 2 year follow up. Eur Arch Psychiatry Clin Neurosci. 2012;262:599-605.

4. Young CM, Findling RL. Pharmacologic treatment of adolescent and child schizophrenia. Expert Rev Neurother. 2004;4:53-60.

5. Hollis C. Adult outcomes of child- and adolescent-onset schizophrenia: diagnostic stability and predictive validity. Am J Psychiatry. 2000;157: 1652-9.

6. Rabinowitz J, Levine SZ, Häfner H. A population based elaboration of the role of age of onset on the course of schizophrenia. Schizophr Res. 2006:88:96-101.

7. James A, Clacey J, Seagroatt V, Goldacre M. Adolescent inpatient psychiatric admission rates and subsequent one-year mortality in England: 1998-2004. J Child Psychol Psychiatry. 2010;51:1395-404.

8. Spencer E, Birchwood M, McGovern D. Management of first-episode psychosis. Adv Psychiatr Treat. 2001;7:133-42.

9. National Institute for Health and Care Excellence. Psychosis and schizophrenia in children and young people: recognition and management (Clinical guideline CG155). UK: NICE; 2013.

10. Stafford MR, Mayo-Wilson E, Loucas CE, James A, Hollis C, Birchwood M, et al. Efficacy and safety of pharmacological and psychological interventions for the treatment of psychosis and schizophrenia in children, adolescents and young adults: a systematic review and metaanalysis. PLoS One. 2015;10:e0117166.

11. Krause M, Zhu Y, Huhn M, Schneider-Thoma J, Bighelli I, Chaimani A, et al. Efficacy, acceptability, and tolerability of antipsychotics in children and adolescents with schizophrenia: a network meta-analysis. Eur Neuropsychopharmacol. 2018;28:659-74.

12. Haddad PM, Sharma SG. Adverse effects of atypical antipsychotics. CNS Drugs. 2007;21:911-36.

13. Correll CU. Assessing and maximizing the safety and tolerability of antipsychotics used in the treatment of children and adolescents. J Clin Psychiatry. 2008:69:26-36.

14. McEvoy JP, Meyer JM, Goff DC, Nasrallah HA, Davis SM, Sullivan L, et al. Prevalence of the metabolic syndrome in patients with schizophrenia: baseline results from the Clinical Antipsychotic Trials of Intervention Effectiveness (CATIE) schizophrenia trial and comparison with national estimates from NHANES III. Schizophr Res. 2005;80:19-32.

15. Correll CU, Manu P, Olshanskiy V, Napolitano B, Kane JM, Malhotra AK. Cardiometabolic risk of second-generation antipsychotic medications during first-time use in children and adolescents. JAMA. 2009;302:1765-73.

16. Perkins DO, Gu H, Weiden PJ, McEvoy JP, Hamer RM, Lieberman JA. Predictors of treatment discontinuation and medication nonadherence in patients recovering from a first episode of schizophrenia, schizophreniform disorder, or schizoaffective disorder: a randomized, double-blind, flexibledose, multicentre study. J Clin Psychiatry. 2008;69:106-13.

17. Jauhar S, McKenna PJ, Radua J, Fung E, Salvador R, Laws KR. Cognitivebehavioural therapy for the symptoms of schizophrenia: systematic review and meta-analysis with examination of potential bias. $\mathrm{Br} J$ Psychiatry. 2014;204:20-9.

18. Wykes T, Steel C, Everitt B, Tarrier N. Cognitive behavior therapy for schizophrenia: effect sizes, clinical models, and methodological rigor. Schizophre Bull. 2008;34:523-37.

19. Bird V, Premkumar P, Kendall T, Whittington C. Early intervention services, cognitive-behavioural therapy and family intervention in early psychosis: systematic review. Br J Psychiatry. 2010;197:350-6.

20. Morrison AP, Turkington D, Pyle M, Spencer H, Brabban A, Dunn G, et al. Cognitive therapy for people with schizophrenia spectrum disorders not taking antipsychotic drugs: a single-blind randomised controlled trial. Lancet. 2014;383:1395-403.

21. Morrison AP, Law H, Carter L, Sellers R, Emsley R, Pyle M, et al. Antipsychotic drugs versus cognitive behavioural therapy versus a combination of both in people with psychosis: a randomised controlled pilot and feasibility study. Lancet Psychiatry. 2018:5:411-23.

22. Morrison AP. The interpretation of intrusions in psychosis: an integrative cognitive approach to hallucinations and delusions. Behav Cogn Psychother. 2001;29:257-76. 
23. Morrison AP. A manualised treatment protocol to guide delivery of evidence-based cognitive therapy for people with distressing psychosis: learning from clinical trials. Psychosis. 2017;9:271-81.

24. Chadwick PD, Birchwood MJ, Trower P. Cognitive therapy for delusions, voices and paranoia. Chichester: Wiley; 1996.

25. Fowler D, Garety P, Kuipers E. Cognitive-behaviour therapy for psychosis: theory and practice. Chichester: Wiley; 1995.

26. Morrison AP, Renton JC, Dunn H, Williams S, Bentall RP. Cognitive therapy for psychosis: a formulation-based approach. London: BrunnerRoutledge; 2004.

27. Fadden G, Birchwood M, Jackson C, Barton K. Psychological therapies: implementation in early intervention services. In: McGorry P, Gleeson J, editors. Psychological interventions in early psychosis: a practical treatment handbook. Chichester: Wiley; 2004. p. 261-80.

28. Blackburn IM, James I, Milne D, Baker CA, Standart S, Garland A, et al. The revised cognitive therapy scale (CTS-R): psychometric properties. Behav Cogn Psychother. 2001;29:431-46.

29. Kay SR, Fiszbein A, Opler LA. The Positive and Negative Syndrome Scale (PANSS) for schizophrenia. Schizophre Bull. 1987;13:261-76.

30. Lecomte T, Corbière M, Ehmann T, Addington J, Abdel-Baki A, MacEwan B. Development and preliminary validation of the First Episode Social Functioning Scale for early psychosis. Psychiatry Res. 2014;216:412-7.

31. Law H, Neil ST, Dunn G, Morrison AP. Psychometric properties of the Questionnaire about the Process of Recovery (QPR). Schizophr Res. 2014;156:184-9.

32. Ronald A, Sieradzka D, Cardno AG, Haworth CMA, McGuire P, Freeman D. Characterization of psychotic experiences in adolescence using the Specific Psychotic Experiences Questionnaire: findings from a study of 5000 16-yearold twins. Schizophre Bull. 2014;40:868-77.

33. Zigmond AS, Snaith RP. The hospital anxiety and depression scale. Acta Psychiatr Scand. 1983;67:361-70.

34. Cassidy CM, Schmitz N, Malla A. Validation of the alcohol use disorders identification test and the drug abuse screening test in first episode psychosis. Can J Psychiatr. 2008;53:26-33.

35. Skinner HA. The drug abuse screening test. Addict Behav. 1982;7:363-71.

36. First MA, Spitzer RL, Gibbon M, Williams JBW. Structured Clinical Interview for DSM-IV Axis I Disorders (SCID-IV). Washington, DC: American Psychiatric Press; 1995.

37. Allison C, Auyeung B, Baron-Cohen S. Toward brief red flags for autism screening: the short Autism Spectrum Quotient and the Short Quantitative Checklist in 1,000 cases and 3,000 controls. J Am Acad Child Adolesc Psychiatry. 2012;51:202-12 e7.

38. Camacho EM, Davies LM, Hann R, Small N, Bower P, Chew-Graham C, Baguely $C$, et al. Long-term clinical and cost-effectiveness of collaborative care (versus usual care) for people with mental-physical multimorbidity: a cluster-randomised trial. Br J Psychiatry. 2018;213:456-63.

39. Morrison AP, Pyle M, Gumley A, Schwannauer M, Turkington D, MacLennan $G$, et al. Cognitive-behavioural therapy for clozapine-resistant schizophrenia: The FOCUS RCT. Health Technol Assess. 2019;23:1-144.

40. Herdman M, Gudex C, Lloyd A, Janssen MF, Kind P, Parkin D, et al, Development and preliminary testing of the new five-level version of EQ-5D (EQ-5D-5L). Qual Life Res. 2011;20:1727-36.

41. Edwards PJ, Roberts I, Clarke MJ, DiGuiseppi C, Wentz R, Kwan I, et al. Methods to increase response to postal and electronic questionnaires. Cochrane Database Syst Rev. 2009;3:MR000008.

42. Pyle M, Norrie J, Schwannaeur M, Kingdon D, Gumley A, Turkington D, et al. Design and protocl for the Focusing on Clozapine Unresponsive Symptoms (FOCUS) trial: a randomised controlled trial. BMC Psychiatry. 2016;16:280

43. Ohlsen R, Williamson R, Yusufi B. Interrater reliability of the Antipsychotic Non-Neurological Side-Effects Rating Scale measured in patients treated with clozapine. J Psychopharmacol. 2008;22:323-9.

44. Browne $\mathrm{RH}$. On the use of a pilot sample for sample size determination. Stat Med. 1995;147:1933-40.

45. Lancaster GA, Dodd S, Williamson PR. Design and analysis of pilot studies: recommendations for good practice. J Eval Clin Pract. 2004;10:307-12.

46. Eldridge $\mathrm{SM}$, Chan CL, Campbell MJ, Bond CM, Hopewell S, Thabane L, et al. CONSORT 2010 statement: extension to randomised pilot and feasibility trials. BMJ. 2016;355:i5239.

47. Pontin E, Peters S, Lobban F, Rogers A, Morriss RK. Enhanced relapse prevention for bipolar disorder: a qualitative investigation of value perceived for service users and care coordinators. Implement Sci. 2009;4:4.
48. Peters S, Pontin E, Lobban F, Morriss R. Involving relatives in relapse prevention for bipolar disorder: a multi-perspective qualitative study of value and barriers. BMC Psychiatry. 2011;11:172.

49. Braun V, Clarke V. Using thematic analysis in psychology. Qual Res Psychol. 2006;3:77-101.

50. NVivo qualitative data analysis software. QSR International Pty Ltd: Version $11 ; 2012$.

\section{Publisher's Note}

Springer Nature remains neutral with regard to jurisdictional claims in published maps and institutional affiliations.
Ready to submit your research? Choose BMC and benefit from:

- fast, convenient online submission

- thorough peer review by experienced researchers in your field

- rapid publication on acceptance

- support for research data, including large and complex data types

- gold Open Access which fosters wider collaboration and increased citations

- maximum visibility for your research: over $100 \mathrm{M}$ website views per year

At $\mathrm{BMC}$, research is always in progress.

Learn more biomedcentral.com/submissions 